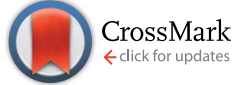

Cite this: Anal. Methods, 2015, 7, 3888

Received 20th February 2015

Accepted 1st April 2015

DOI: $10.1039 / \mathrm{c} 5 \mathrm{ay} 00464 \mathrm{k}$

www.rsc.org/methods

\title{
A fluorescein labeled aptamer switch for thrombin with fluorescence decrease response $\uparrow$
}

\author{
Lihua Hao and Qiang Zhao*ab
}

We developed a simple fluorescent switch for rapid thrombin detection by using a high-binding affinity 27-mer aptamer with fluorescein (FAM) labeled at the $3^{\prime}$ end. This FAM-labeled aptamer showed remarkable fluorescence quenching upon thrombin binding. This assay enabled the detection of thrombin ranging from $0.062 \mathrm{nM}$ to $2 \mathrm{nM}$. The assay was selective, and other tested proteins and diluted serum did not interfere with thrombin analysis.

\section{Introduction}

Aptamers are single-stranded DNA or RNA molecules isolated from a random nucleic acid library through the systematic evolution of ligands by the exponential enrichment (SELEX) process. ${ }^{\mathbf{1 - 4}}$ As alternatives to immunoantibodies, aptamers have great features, such as good stability, facile production, easy labeling with functional groups, and remarkable specificity and affinity for various target molecules. These features make aptamers promising in sensor design and assay development., One of the unique properties of aptamers is that target-binding usually can induce conformational changes, which provides an attractive approach to construct aptamer-based electrochemical and optical assays and sensors. ${ }^{4-6}$

Human $\alpha$-thrombin, a trypsin-like serine protease involved in many physiological and pathological processes, plays an essential role in pulmonary metastasis, diseases associated with coagulation abnormalities, and synovial inflammation as a therapeutic drug and a biomarker for diagnosis of some diseases. $^{7}$ Benefiting from aptamers against thrombin, numerous aptamer-based assays and biosensors with different formats have been developed for thrombin. ${ }^{6}$ A 15-mer aptamer ( $5^{\prime}$-GGT TGG TGT GGT TGG-3' $)^{8}$ binding with the fibrinogen binding site of thrombin and a 29-mer aptamer (5'-AGT CCG TGG TAG GGC AGG TTG GGG TGA CT- $\left.3^{\prime}\right)^{\mathbf{8}, \mathbf{9}}$ binding with the heparin-binding site of thrombin are the mostly used DNA aptamers. The 15-mer aptamer shows a weaker binding affinity to thrombin with a dissociation constant $\left(K_{\mathrm{d}}\right)$ of around $100 \mathrm{nM}$,

anstitute of Environmental Science, College of Chemistry and Chemical Engineering, Shanxi University, Taiyuan, 030006, P. R. China.E-mail: chemzhaoq@hotmail.com; Fax: +86-351-7011011; Tel: +86-351-7011011

${ }^{b}$ State Key Laboratory of Environmental Chemistry and Ecotoxicology, Research Center for Eco-Environmental Sciences, Chinese Academy of Sciences, Beijing, 100085, P. R. China

$\dagger$ Electronic supplementary information (ESI) available: Fig. S1-S3; Tables S1 and S2. See DOI: 10.1039/c5ay00464k while the 29-mer aptamer binds to thrombin with a strong binding affinity to thrombin, showing a $K_{\mathrm{d}}$ of about $0.5 \mathrm{nM} .^{8,9}$

A few fluorescent switches have been reported for thrombin based on binding-induced fluorescence changes by using aptamers labeled with a single dye or two dyes. ${ }^{3,6}$ Li et al. built an aptamer beacon with a fluorophore and a quencher tagged at the $5^{\prime}$-end and $3^{\prime}$-end of the 15 -mer thrombin-binding aptamer. ${ }^{10}$ In the presence of thrombin, the aptamer structure was transferred from random coil to G-quadruplex, resulting in fluorescence quenching due to the proximity between the fluorophore and the quencher. A molecular beacon with a signalincrease response for thrombin detection by using a 15-mer aptamer has also been reported. ${ }^{\mathbf{1 1 , 1 2}}$ Wang et al. developed a simple fluorescent switch for thrombin by using a 15-mer aptamer with tetramethylrhodamine (TMR)-labeled at the end. ${ }^{13}$ In the absence of thrombin, the guanine base was close to TMR, causing fluorescence quenching, while thrombin binding induced a structural change of the aptamer and separated the fluorophore from the guanine bases, leading to the recovery of the fluorescence signal. Most of these approaches used the 15-mer aptamer with a weaker binding affinity or required an expensive dual-labeled aptamer. Previously, we introduced pyrene at both terminals of a 27-mer aptamer (5'-GT CCG TGG TAG GGC AGG TTG GGG TGA C-3') and developed a bindinginduced fluorescent switch for thrombin analysis, which was obtained by truncating one pair of complementary bases from the stem terminal of the 29-mer aptamer against thrombin. In the absence of thrombin, the $5^{\prime}$-end and the $3^{\prime}$-end of the 27-mer aptamer were spatially separated, and upon thrombin binding, the aptamer brought the two ends close to form an excimer of pyrene, giving fluorescence. ${ }^{14}$

In this work, inspired by the capability of binding-induced structural changes of the 27-mer aptamer against thrombin, we developed a simple aptamer fluorescent switch for thrombin analysis with single fluorescein (FAM) labeled at the $3^{\prime}$ end of the 27-mer aptamer. When thrombin binds with the FAMlabeled aptamer, the aptamer probe generates a fluorescence 
quenching response. This assay is simple and rapid for direct analysis of targets without requiring immobilization or washing steps. We achieved detection of thrombin at $62 \mathrm{pM}$, and thrombin could be analyzed in diluted human serum by measuring the change of the fluorescence intensity of the FAM labeled aptamer.

\section{Experimental}

\section{Reagents}

Human transferrin, bovine serum albumin (BSA), hemoglobin (from bovine blood), human immunoglobulin G (IgG) and trypsin (from bovine pancreas) were purchased from Sigma. Proteinase $\mathrm{K}$ was ordered from Merck. Porcine pancreatic elastase and chymotrypsin were obtained from Ruibio. Human $\alpha$-thrombin was purchased from Haematologic Technologies Inc (Essex Junction, VT). The pooled human serum was obtained from Zhongke Chenyu Biotechnology in Beijing. The 27-mer DNA aptamer recognizing thrombin with a FAM dye labeled at the $3^{\prime}$-end had the following sequence: $5^{\prime}$-GTC CGT GGT AGG GCA GGT TGG GGT GAC-3' ${ }^{\prime}{ }^{9}$ denoted as Th27-3-FAM. DNA aptamers without labelling and DNA aptamers with other fluorophore labels (e.g. tetramethylrhodamine (TMR) and Cy3) at different labeling sites are listed in Table S1. $\dagger$ All DNA oligos were synthesized and purified by Sangon Biotech (Shanghai, China). Other reagents used in the experiment were of analytical grade and supplied by Amresco (Solon, OH) or Beijing Chemical Reagents (Beijing, China). Ultra-pure water used in this experiment was obtained through a Purelab Ultra Elga Labwater system. The assay buffer was $20 \mathrm{mM}$ Tris- $\mathrm{HCl}, 10 \mathrm{mM} \mathrm{NaCl}$, $0.5 \mathrm{mM} \mathrm{MgCl}_{2}$, and $0.5 \mathrm{~g} \mathrm{~L}^{-1} \mathrm{BSA}$ (pH 7.5).

\section{Detection of thrombin}

The detection of thrombin was performed under optimized experimental conditions. All fluorescence measurements were performed on a JASCO FP-8300 fluorescence spectrometer (Japan) at $25{ }^{\circ} \mathrm{C}$. For experiments measuring the fluorescence intensity of FAM-labeled probes, the excitation wavelength was set at $498 \mathrm{~nm}$ and the fluorescence spectra from 510 to $600 \mathrm{~nm}$ were recorded. The fluorescence intensity of the TMR labeled aptamer with an excitation of $560 \mathrm{~nm}$ was recorded from 570 to $650 \mathrm{~nm}$ and the fluorescence intensity of the Cy3 labeled aptamer with an excitation of $550 \mathrm{~nm}$ was recorded from 562 to $650 \mathrm{~nm}$. Slits for both excitation and emission were set at $5 \mathrm{~nm}$. In the assay for thrombin using Th27-3-FAM, solution $(120 \mu \mathrm{L})$ containing $20 \mathrm{mM}$ Tris- $\mathrm{HCl}(\mathrm{pH} 7.5), 10 \mathrm{mM} \mathrm{NaCl}, 0.5 \mathrm{mM}$ $\mathrm{MgCl}_{2}, 0.5 \mathrm{~g} \mathrm{~L}^{-1} \mathrm{BSA}, 2 \mathrm{nM}$ aptamer probes, and thrombin at different concentrations was tested by monitoring the fluorescence intensity changes at $518 \mathrm{~nm}$.

\section{Specificity test}

To test the specificity of the aptamer for thrombin, other proteins (hemoglobin, lysozyme, human immunoglobulin G, proteinase $\mathrm{K}$, trypsin, chymotrypsin, and porcine pancreatic elastase) were analyzed by using the same procedure described above for the detection of thrombin standard with Th27-3-FAM.
In the specificity analysis, $120 \mu \mathrm{L}$ of sample containing $2 \mathrm{nM}$ aptamer probes and thrombin $(8 \mathrm{nM})$ or other proteins $(16 \mathrm{nM})$ were tested.

To determine whether the assay could be used for the detection of thrombin in a complex matrix, thrombin spiked in 250 -fold diluted human serum was analyzed. Thrombin at varying concentrations was tested with Th27-3-FAM.

\section{Results and discussion}

\section{Assay feasibility and choice of the aptamer probe}

Fig. 1 shows the schematic diagram of the detection of thrombin using the 27-mer thrombin-binding aptamer with FAM labeled at the 3 -end. The binding of thrombin to the aptamer causes the structural change of the aptamer, ${ }^{14}$ and the fluorescence intensity of the labeled FAM is quenched due to possible changes in the local environment of FAM (e.g. proximity to bases of the aptamer or the bound thrombin) or its possible interaction with bases like $G$ of the aptamer ${ }^{13}$ or thrombin. We first tested the feasibility of the assay for thrombin by using the 27-mer aptamer with FAM labeled at the $3^{\prime}$ end (Th27-3-FAM). Fig. 2 shows the spectra of Th27-3-FAM in the blank solution without thrombin and the solution containing thrombin. In the presence of thrombin, the Th27-3-FAM probe showed an obvious fluorescence decrease at the wavelength of $518 \mathrm{~nm}$. This result suggests that the assay for thrombin using Th27-3-FAM is feasible.

To investigate whether dye types affect the fluorescence response of the fluorescent switch, we tested the 27-mer aptamer with TMR labeled at the $3^{\prime}$ end (Th27-3-TMR) or Cy3 labeled at the $3^{\prime}$ end (Th27-3-Cy3) (Fig. 3). Th27-3-TMR showed a lower fluorescence intensity as TMR was easily quenched by aptamer bases. ${ }^{13}$ Both Th27-3-TMR and Th27-3-Cy3 could show a fluorescence decrease response upon thrombin, but the obtained response was lower than that from Th27-3-FAM. When FAM was labeled at the $5^{\prime}$ end of the 27-mer aptamer (Th27-5-FAM), a smaller change of the fluorescence intensity in the presence of thrombin was observed than that obtained by Th27-3-FAM, showing that the $3^{\prime}$ end was favourable for FAM labeling to give a better response. This result may be explained by the bindinginduced closeness of FAM to G bases on the other end of Th273-FAM, and G could cause fluorescence quenching. ${ }^{13}$ In contrast, in Th27-5-FAM, FAM was labeled at the 5'end of the aptamer, already close to a $\mathrm{G}$ base, so the fluorescence intensity of FAM

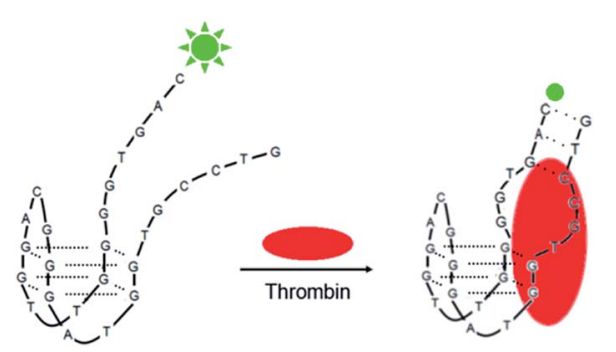

Fig. 1 Schematic diagram of the detection of thrombin by using the 27-mer aptamer with FAM-labeled at the $3^{\prime}$ end. 


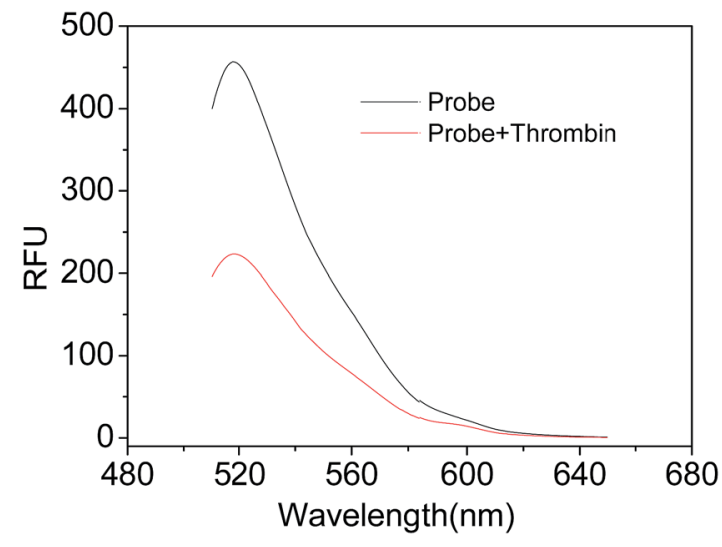

Fig. 2 Emission spectra of the Th27-3-FAM in the absence and presence of thrombin. RFU: relative fluorescence unit. The buffer solution was $20 \mathrm{mM}$ Tris- $\mathrm{HCl}, 10 \mathrm{mM} \mathrm{NaCl}, 0.5 \mathrm{mM} \mathrm{MgCl}_{2}$, and $0.5 \mathrm{~g} \mathrm{~L}^{-1} \mathrm{BSA}$ ( $\mathrm{pH}$ 7.5). The concentration of probes was $2 \mathrm{nM}$ and the concentration of thrombin was $8.3 \mathrm{nM}$.

was lower and the binding-induced fluorescence change was smaller. Instead of the 27-mer aptamer, the 29-mer aptamer with FAM labeled at the $3^{\prime}$ end (Th29-3-FAM) was also tested. Th293-FAM showed a decreased fluorescence intensity in the absence of thrombin and a smaller intensity change induced by thrombin, which might be attributed to the fact that the 29-mer aptamer already had a more stable duplex structure at the end in the absence of thrombin as shown in previous studies. ${ }^{\mathbf{9 1 4}}$ Circular dichroism (CD) experiments show no difference in the spectra for the 27-mer aptamer (Th27) and the 29-mer aptamer (Th29) in the absence of thrombin (Fig. S1 $\dagger$ ). The addition of thrombin caused a significant change of CD spectra for both Th29 and Th27 (Fig. S1 $\dagger$ ). CD experiments show that thrombin addition can cause structural changes of aptamers. Compared with responses of other tested aptamer probes (Th27-5-FAM, Th27-3-TMR and Th27-3-Cy3, and Th29-3-FAM) to thrombin, Th27-3-FAM exhibited a higher fluorescence response to thrombin. The results suggest that the dye species, labeling sites and the length of the stem of the aptamer have a great effect on the construction of fluorescent switches for thrombin. Th273-FAM was used in this work for the detection of thrombin. However, the detailed mechanism for Th27-3-FAM to generate fluorescence quenching for thrombin binding and the reasons for Th27-3-FAM to give better responses than other dye-labeled aptamer probes are not clear yet (Fig. 3).

\section{Optimization of experimental conditions}

To obtain a good fluorescence response to thrombin with Th273-FAM, we further optimized the experimental conditions. The influence of sodium ions on the experiment was investigated first. As shown in Fig. 4, when there was no $\mathrm{NaCl}$ in the binding buffer solution, the fluorescence decrease induced by thrombin was minimal, which might be related to the fact that a low concentration of $\mathrm{NaCl}$ was not good for stabilizing the conformation of aptamer probes and maintaining a good binding affinity. ${ }^{\mathbf{1 4}}$ The obtained absolute fluorescence response increased when $10 \mathrm{mM} \mathrm{NaCl}$ was present in the binding buffer

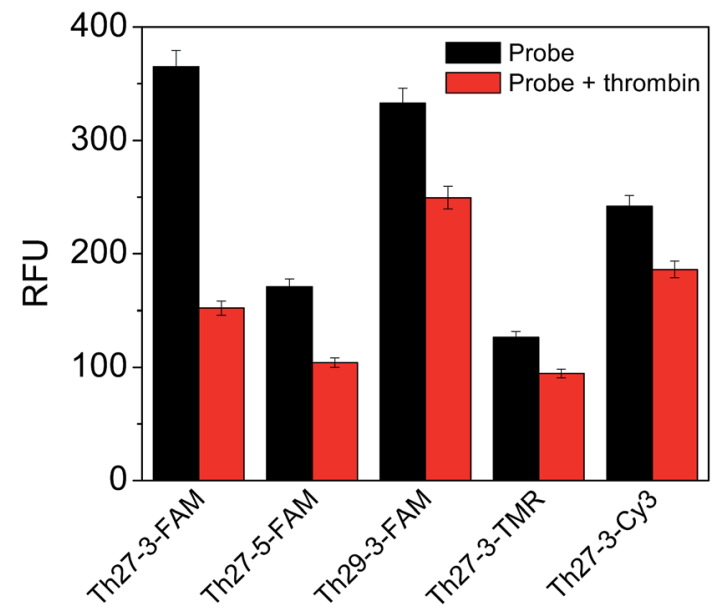

Fig. 3 Fluorescence intensity of aptamer probes before and after adding thrombin. The buffer solution was $20 \mathrm{mM}$ Tris- $\mathrm{HCl}, 10 \mathrm{mM}$ $\mathrm{NaCl}, 0.5 \mathrm{mM} \mathrm{MgCl}_{2}$, and $0.5 \mathrm{~g} \mathrm{~L}^{-1} \mathrm{BSA}$ ( $\mathrm{pH}$ 7.5). The concentration of all probes was $2 \mathrm{nM}$ and the concentration of thrombin was $8.3 \mathrm{nM}$.

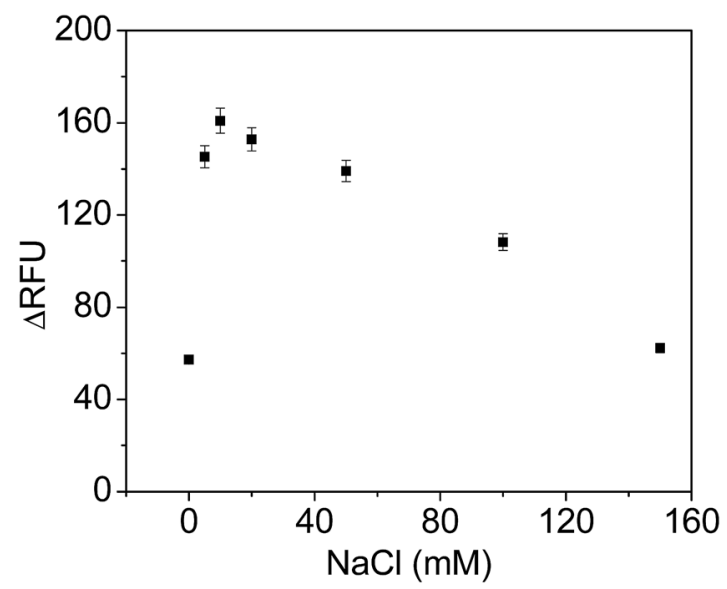

Fig. 4 Effect of $\mathrm{Na}^{+}$concentration on the fluorescence change of the aptamer caused by thrombin in the binding solution containing $20 \mathrm{mM}$ Tris- $\mathrm{HCl}(\mathrm{pH}$ 7.5), $2 \mathrm{nM}$ aptamer probes, $8.3 \mathrm{nM}$ thrombin and varying concentrations of $\mathrm{NaCl}$.

solution, and then the responses decreased with further addition of $\mathrm{NaCl}$, which might result from the fact that a high concentration of $\mathrm{NaCl}$ could weaken the attractive electrostatic interaction and reduce the binding affinity. ${ }^{\mathbf{1 4}}$ Therefore, in this experiment the buffer containing $10 \mathrm{mM} \mathrm{NaCl}$ was used for the quantitative detection of thrombin. Then, we examined the influence of $\mathrm{MgCl}_{2}$. When binding buffer contained $0.5 \mathrm{mM}$ of $\mathrm{MgCl}_{2}$, Th27-3-FAM could give higher responses to thrombin (Fig. S2(A) †). BSA was used to reduce possible nonspecific interactions of aptamer probes and thrombin on the surface of tubes. The influence of BSA on the experiment was investigated. As shown in Fig. S2(B), $\dagger$ BSA at $0.5 \mathrm{~g} \mathrm{~L}^{-1}$ caused a larger change of fluorescence intensity of the Th27-3-FAM probe, suggesting that BSA may favour the binding-induced structural change of aptamers and help aptamers bind to thrombin. Therefore, the buffer with $20 \mathrm{mM}$ Tris- $\mathrm{HCl}, 10 \mathrm{mM} \mathrm{NaCl}, 0.5 \mathrm{mM} \mathrm{MgCl}_{2}$, and $0.5 \mathrm{~g} \mathrm{~L}^{-1} \mathrm{BSA}(\mathrm{pH}$ 7.5) was used in the further study. 


\section{Detection of thrombin}

Thrombin detection was achieved with a Th27-3-FAM aptamer under optimized conditions. As shown in Fig. 5, upon the addition of thrombin, the fluorescence intensity at $518 \mathrm{~nm}$ decreased with an increase of thrombin, and when the thrombin concentration was higher than $4 \mathrm{nM}$, the fluorescence intensity did not further decrease. Thrombin ranging from $0.062 \mathrm{nM}$ to $2 \mathrm{nM}$ could be detected with a good linear correlation $\left(y=96.86 x+0.57, R^{2}=0.997\right)$ between the absolute fluorescence change and the thrombin concentration. The detection limit of thrombin was $0.062 \mathrm{nM}(S / N=3)$. Compared with a few reported aptamer-based fluorescence assays for thrombin, this assay has more simple steps and a lower limit (Table S2†). ${ }^{10,11,13,15-18}$

\section{Specificity test}

To assess the selectivity of the probe, we tested a few blood proteins and structurally similar proteins to thrombin such as hemoglobin, lysozyme, human immunoglobulin $\mathrm{G}$, proteinase $\mathrm{K}$, trypsin, chymotrypsin, and porcine pancreatic elastase. As shown in Fig. 6, other proteins did not cause apparent
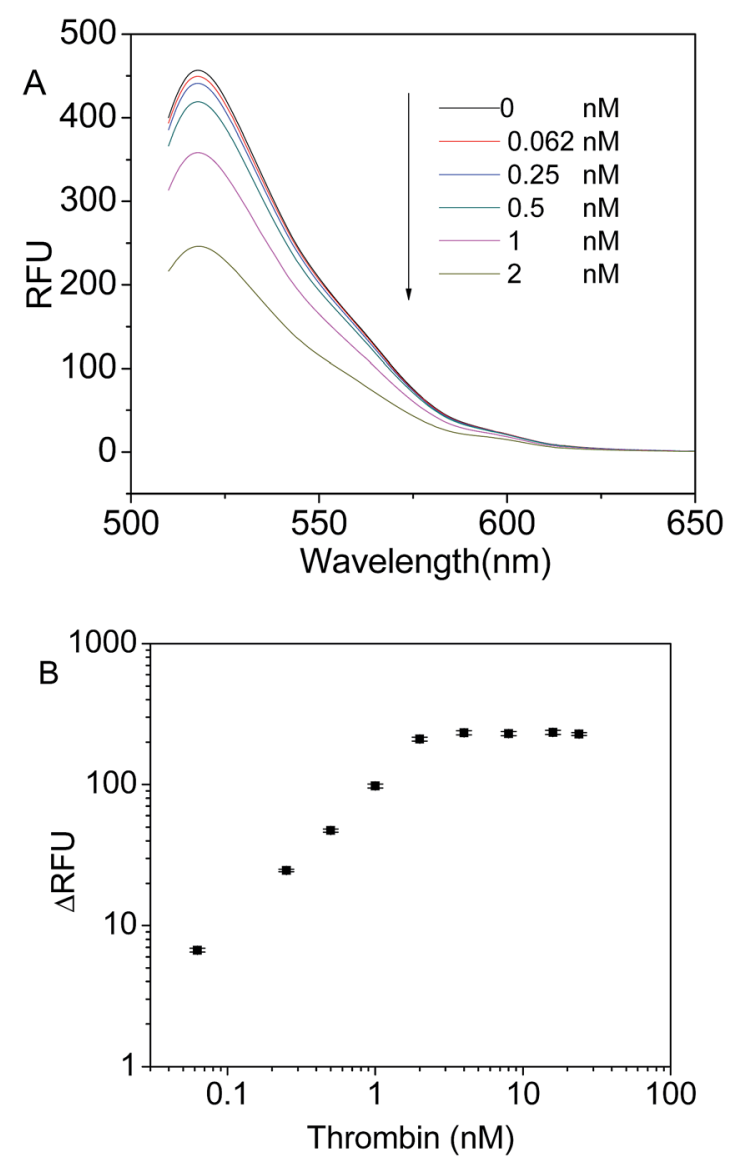

Fig. 5 Detection of thrombin with the Th27-3-FAM aptamer probe. (A) Emission spectra of aptamer probes with increasing concentrations of thrombin. (B) Absolute fluorescence change caused by varying concentrations of thrombin.

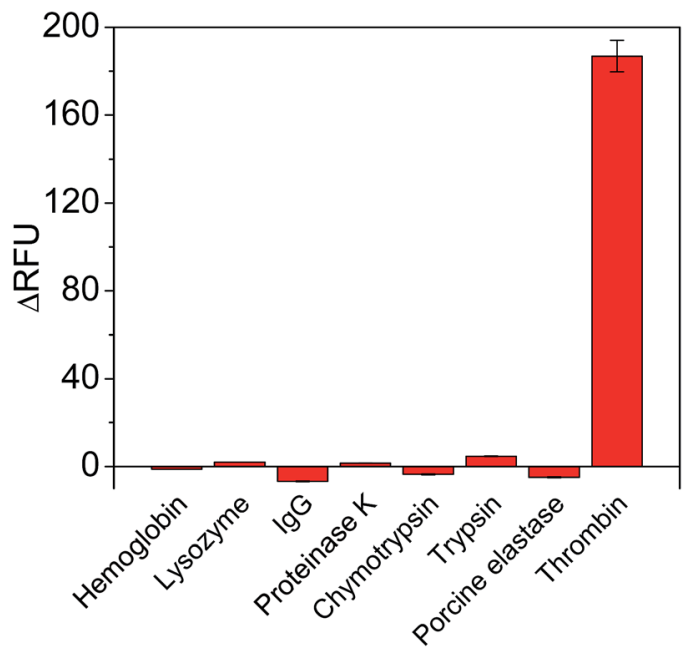

Fig. 6 The specificity of the assay. The concentration of thrombin and the other tested proteins were $8 \mathrm{nM}$ and $16 \mathrm{nM}$, respectively.

fluorescence intensity changes of aptamer probes. The results show that the fluorescent switch is selective.

To further test the selectivity of the assay for thrombin in a complex sample matrix, we took diluted human serum as an example. Thrombin was spiked into 250-fold diluted human serum and was analyzed. Fig. S3† shows that thrombin in the diluted serum sample could be detected in the range of 0.125 to $2 \mathrm{nM}$ with a linear correlation $\left(y=74.45 x+2.39, R^{2}=0.991\right)$ between the fluorescence change and thrombin concentration, which was achieved with a detection limit of $0.125 \mathrm{nM}(S / N=3)$.

\section{Conclusions}

In summary, a simple, sensitive and economical method for rapid thrombin detection by using a FAM labeled 27-mer aptamer against thrombin was reported. The FAM labeled aptamer showed an obvious fluorescence decrease upon thrombin binding. $62 \mathrm{pM}$ thrombin could be detected with this simple fluorescent switch, and thrombin could be analyzed in a diluted serum sample. This 27-mer thrombin aptamer can be used to develop sensors with other detection formats for thrombin analysis.

\section{Acknowledgements}

This work was supported by the National Natural Science Foundation of China (Grant no. 21222503), the Outstanding Youth Talents Program of Shanxi Province, and the Key Project of Chinese Ministry of Education (Grant no. 212020).

\section{Notes and references}

1 A. D. Ellington and J. W. Szostak, Nature, 1990, 346, 818.

2 C. Tuerk and L. Gold, Science, 1990, 249, 505.

3 B. Juskowiak, Anal. Bioanal. Chem., 2011, 399, 3157.

4 E. J. Cho, J.-W. Lee and A. D. Ellington, Annu. Rev. Anal. Chem., 2009, 2, 241. 
5 M. Citartan, S. C. B. Gopinath, J. Tominaga, S.-C. Tan and T.-H. Tang, Biosens. Bioelectron., 2012, 34, 1.

6 B. Deng, Y. Lin, C. Wang, F. Li, Z. Wang, H. Zhang, X.-F. Li and X. C. Le, Anal. Chim. Acta, 2014, 837, 1.

7 Thrombin: Physiology and Disease, ed. M. E. Maragoudakis and N. E. Tsopanoglou, Springer Science, New York, 2009.

8 L. C. Bock, L. C. Griffin, J. A. Latham, E. H. Vermaas and J. J. Toole, Nature, 1992, 55, 564.

9 D. M. Tasset, M. F. Kubik and W. Steiner, J. Mol. Biol., 1997, 272, 688.

10 J. J. Li, X. H. Fang and W. H. Tan, Biochem. Biophys. Res. Commun., 2002, 292, 31.

11 N. Hamaguchi, A. Ellington and M. Stanton, Anal. Biochem., 2001, 294, 126.
12 Z. W. Tang, P. Mallikaratchy, R. Yang, Y. Kim, Z. Zhu, H. Wang and W. H. Tan, J. Am. Chem. Soc., 2008, 130, 11268.

13 W. J. Wang, C. L. Chen, M. X. Qian and X. S. Zhao, Sens. Actuators, B, 2008, 129, 211.

14 Q. Zhao and L. Cheng, Anal. Bioanal. Chem., 2013, 405, 8233. 15 Q. L. Yue, T. F. Shen, L. Wang, S. L. Xu, H. B. Li, Q. W. Xue, Y. F. Zhang, X. H. Gu, S. Q. Zhang and J. F. Liu, Biosens. Bioelectron., 2014, 56, 231.

16 B. L. Li, H. Wei and S. J. Dong, Chem. Commun., 2007, 73.

17 D. W. Huang, C. G. Niu, Z. Z. Li, M. Ruan, X. Y. Wang and G. M. Zeng, Analyst, 2012, 137, 5607.

18 E. Katilius, Z. Katiliene and N. W. Woodbury, Anal. Chem., 2006, 78, 6484. 